PROCEEDINGS OF THE

AMERICAN MATHEMATICAL SOCIETY

Volume 130, Number 12, Pages 3745-3746

S 0002-9939(02)06746-1

Article electronically published on June 27, 2002

\title{
ON ANTI-AUTOMORPHISMS OF THE FIRST KIND IN DIVISION RINGS
}

\author{
PETER BEELEN AND RALF GRAMLICH
}

(Communicated by Martin Lorenz)

\begin{abstract}
We provide a short proof of the well-known fact that a division ring of finite degree over its center that admits an anti-automorphism of the first kind, i.e., an anti-automorphism that fixes the center elementwise, also admits an involutive anti-automorphism.
\end{abstract}

Albert has shown that a division ring of finite degree over its center that admits an anti-automorphism of the first kind also admits an involutive anti-automorphism; cf. Theorem X.19 of [1. A proof of this theorem can now be found in a number of textbooks, e.g. [2], [3], [4], [5], [6], or [7]. The purpose of this note is to give another short proof.

Definition 1. A finite dimensional central simple algebra $A$ is called a crossed product if $A$ has a maximal subfield $\mathbb{K}$ which is Galois over the center of $A$.

Theorem 2. Let $A$ be a crossed product and let $\mathbb{F}$ be the center of $A$. Suppose that $A$ allows an anti-automorphism that fixes $\mathbb{F}$ elementwise. Then $A$ allows an involutive anti-automorphism.

Proof. Let $\sigma: A \rightarrow A$ be an anti-automorphism that fixes $\mathbb{F}$ elementwise. Since $A$ is a crossed product, there exists a maximal subfield $\mathbb{K}$ of $A$ such that $\mathbb{K} / \mathbb{F}$ is Galois. Denote the corresponding Galois group by $G$. By Theorem 3.1.27 of [ 5 ] for every $g \in G$ there exists an element $j_{g} \in A$ with $g(k)=j_{g} k j_{g}^{-1}$ for all $k \in \mathbb{K}$, such that $A=\bigoplus_{g \in G} \mathbb{K} j_{g}$. Using the Skolem-Noether theorem, we can assume that $\sigma$ fixes $\mathbb{K}$ elementwise and so for every $g \in G$ we have $j_{g} k j_{g}^{-1}=g(k)=$ $\sigma(g(k))=\sigma\left(j_{g} k j_{g}^{-1}\right)=\sigma\left(j_{g}\right)^{-1} k \sigma\left(j_{g}\right)$. Therefore $\sigma\left(j_{g}\right) j_{g} \in C_{A}(\mathbb{K})=\mathbb{K}$. Hence $\sigma\left(j_{g}\right)=a_{g} j_{g}^{-1}$ for some $a_{g} \in \mathbb{K}^{*}$. This implies $\sigma^{2}\left(j_{g}\right)=\sigma\left(j_{g}^{-1}\right) a_{g}=j_{g}$, and thus $\sigma^{2}$ is the identity.

Corollary 3. Let $\mathbb{D}$ be a division ring of finite degree over its center $\mathbb{F}$. Suppose that $\mathbb{D}$ allows an anti-automorphism that fixes $\mathbb{F}$ elementwise. Then $\mathbb{D}$ allows an involutive anti-automorphism.

Proof. There exists an $n \in \mathbb{N}$ such that $M_{n}(\mathbb{D})$ is a crossed product by Theorem V.1 of $\left[1\right.$. We can extend $\sigma$ to an anti-automorphism $\bar{\sigma}$ of $M_{n}(\mathbb{D})=\mathbb{D} \otimes_{\mathbb{F}} M_{n}(\mathbb{F})$ by letting $\bar{\sigma}$ act as the transposition map on $1 \otimes M_{n}(\mathbb{F})$. Theorem 2 implies that

Received by the editors December 7, 2001 and, in revised form, February 20, 2002.

2000 Mathematics Subject Classification. Primary 16K20, 16W99, 51A10, 51A30.

Key words and phrases. Division ring, anti-automorphism.

(C)2002 American Mathematical Society 
there exists an involutive anti-automorphism on $M_{n}(\mathbb{D})$. By Theorem 3.1.70 of [5], we conclude that $\mathbb{D}$ allows an involutive anti-automorphism.

\section{ACKNOWLEDGMENT}

The authors would like to express their gratitude to Zinovy Reichstein for pointing out a generalization of their original proof.

\section{REFERENCES}

[1] A.A. Albert, Structure of algebras, American Mathematical Society, New York 1939. MR $1: 99 \mathrm{c}$

[2] P.K. Draxl, Skew fields, London Math. Soc. Lecture Note Series 81, Cambridge University Press, Cambridge 1983. MR 85a:16022

[3] N. Jacobson, Finite-dimensional division algebras over fields, Springer, Berlin 1996. MR 98a:16024

[4] M.-A. Knus, A. Merkurjev, M. Rost, J.-P. Tignol, The Book of Involutions, AMS Colloquium Publications 44, 1998. MR 2000a:16031

[5] L.H. Rowen, Polynomial identities in ring theory, Academic Press, New York 1980. MR 82a:16021

[6] D.J. Saltman, Azumaya algebras with involution, J. Algebra 53 (1978), pp. 526-539. MR 80a:16013

[7] W. Scharlau, Quadratic and Hermitian forms, Springer-Verlag, Berlin 1985. MR 86k:11022

Department of Mathematics and Computer Science, TU Eindhoven, P.O. Box 513, 5600 MB Eindhoven, The Netherlands

E-mail address: pbeelen@win.tue.nl

Department of Mathematics and Computer Science, TU Eindhoven, P.O. Box 513, 5600 MB Eindhoven, The Netherlands

E-mail address: squid@win.tue.nl 